\title{
端 Sovilo \\ Análisis \\ Violencia hacia las mujeres: recuperando la voz de la mujer indígena
}

\author{
María Engracia Carrazco Valenzuela* \\ Josefina Pérez Martínez**
}

\section{Resumen}

El contenido del artículo tiene como finalidad esbozar un planteamiento donde se dimensionan elementos conceptuales y contextuales sobre el problema de las violencias y particularmente de la violencia de género hacia las mujeres indígenas.
La violencia hacia las mujeres ha ido en aumento y no es privativo de un tipo de comunidad en lo específico, es un asunto que trastoca el ámbito familiar, social y cultural, fundamentalmente.

Recurrir a los resultados de estudios e investigaciones en materia de violencia, permite procesos

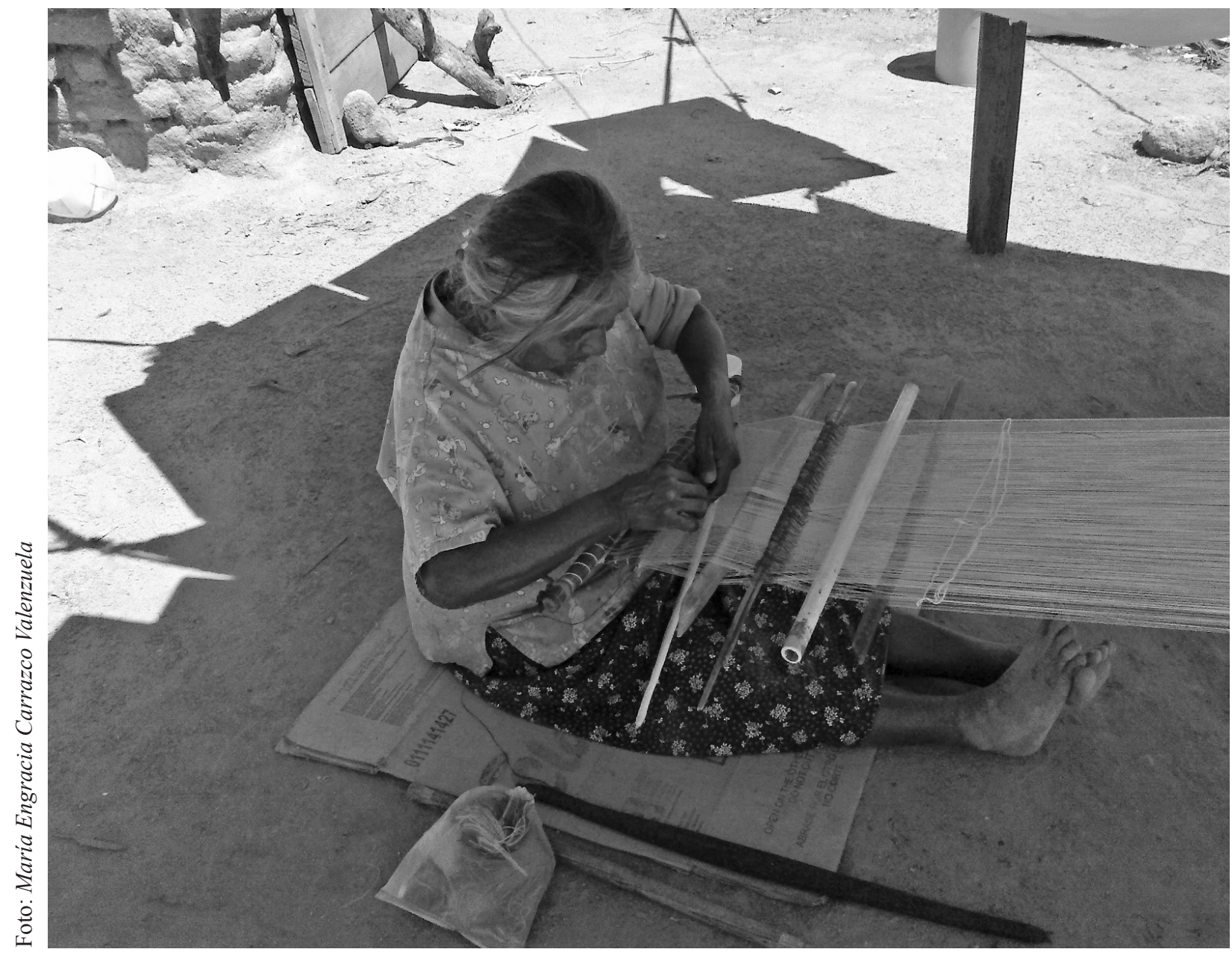

* Licenciada en Trabajo Social, Maestra en Innovación Educativa por la Universidad de Sonora, Profesora investigadora en el Departamento de Trabajo Social de la Universidad de Sonora. mcarrazco@sociales.uson.mx

${ }^{* *}$ Alumna del noveno semestre del programa de licenciatura en Trabajo Social de la Universidad de Sonora. josy1706@hotmail.com 


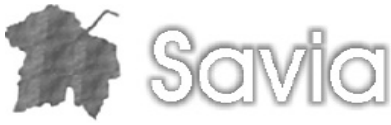

de reflexión, comprensión y análisis del problema, toda vez que aportan mayores elementos para la interpretación y la posibilidad de repensar en el diseño de estrategias que puedan contribuir a la definición de acciones para la prevención y la erradicación de un problema tan complejo que cada vez se agudiza en la sociedad mexicana.

Se recupera la voz de la mujer indígena a través de la entrevista, que se constituye en una técnica que posibilita rescatar el pensar, sentir y decir de una mujer indígena triqui, que desde su perspectiva cultural y de los usos y costumbres del entorno en el que se desarrolla cotidianamente, percibe el problema y le asigna su propia interpretación.

\section{Abstract}

The content of this article emphasizes the conceptual and contextual elements against violence, especially violence against indigenous women.

Violence against women is rising and it is not restricted to certain type of community, it is a matter that covers every family circle, society and culture.

Referring to some results of studies and investigations related with violence, let us reflect, comprehend and analyze this problem; as long as those results provide greater elements to interpret and the possibility to rethink about the design of strategies that could contribute to the definition of actions for the prevention and eradication of such a complex problem which is getting more consistent among Mexican society.

Throughout this interview, indigenous women have been heard, such is the case of a Triqui woman who is aware of this problem but because of her cultural perspective and daily customs, has her own interpretation of it.

\section{Introducción}

Hoy en día se habla mucho del problema de violencia en todas sus dimensiones: violencia intrafamiliar, hacia las niñas y niños, escolar, estructural, de género, entre otras formas de violencia.

Cuando se refiere al concepto de violencia, se establece una relación directa con todas aquellas formas que de una manera u otra generan exclusión, discriminación, agresión y maltrato; son manifestaciones que llevan a un estado de vulnerabilidad social que sin duda afectan la dignidad e integridad de las personas.

Dichas manifestaciones han sido consideradas históricamente como resultado de la diversidad de problemas de orden social, político, económico, cultural, religioso y étnico, entre otros; sus causas son complejas en tanto se identifican con factores asociados a los contextos en los que se desarrollan los individuos, grupos y colectividades más vulnerables de la población.

Pensar y repensar sobre la condición de la mujer, específicamente la mujer indígena, la cual es víctima de violencia en una sociedad donde los patrones culturales determinan que se sigan preservando muchas de las tradiciones que durante el tiempo se han venido trasmitiendo de generación en generación. Ello, de alguna manera coloca a la mujer en una situación de desventaja, y a la mujer indígena aún más por su propia condición; es decir, ser mujer y ser indígena; ya que se ha visto obligada a silenciar lo que le sucede en su mundo en donde las formas de violencia atentan contra sus propios derechos.

Es oportuno decir que es necesario abordar desde los diferentes espacios, la reflexión y discusión sobre la cuestión de la violencia de la mujer, y que ello conlleve a construir propuestas para el impulso de programas y proyectos sociales de atención a las mujeres indígenas que se encuentran, sobre todo en todas aquellas comunidades alejadas de algunos de los beneficios institucionales que pretendan contribuir a disminuir las distintas formas de violencia que se asumen y que se ejercen contra las mujeres.

Este ejercicio de reflexión, pretende recuperar y exponer un breve planteamiento sobre algunos postulados que desde los diferentes ámbitos se han abordado sobre la violencia hacia las mujeres como un fenómeno social; también pretende recuperar parte de la voz de quienes han vivido experiencias que trascienden en la vida cotidiana de la mujer indígena en un espacio y tiempo determinado. 


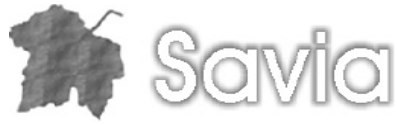

\section{Desarrollo}

Para sustentar lo anterior, se abordan algunas conceptualizaciones generales sobre la violencia, las cuales permiten dimensionar que se trata de un problema que viven las mujeres en el que existen muchos vacíos por enfrentar y resolver.

La exclusión y discriminación social, en la relación que explícita e implícitamente guardan, pueden generar un nuevo escenario; este, se define como las diversas formas de expresión de las violencias.

Si bien existen una serie de definiciones sobre violencia, se recurre a la planteada por la Organización Mundial de la Salud (OMS): "EI uso deliberado de la fuerza física o el poder, ya sea en grado de amenaza o efectivo, contra uno mismo, otra persona o un grupo o comunidad, que cause o tenga muchas probabilidades de causar lesiones, muerte, daños psicológicos, trastornos del desarrollo o privaciones" (Organización Panamericana de la Salud, 2002).

La Real Academia Española define la condición de "violento" en muy diversos sentidos, entre estos se incluyen "estar fuera de su natural estado", obrar "con ímpetu y fuerza", hacer "con intensidad extraordinaria" y ejecutar "contra el modo regular o fuera de razón y justicia". La etimología de "violencia" indica que es un vocablo tomado del latín violentĭa y violentus, derivado de vis, que significa "fuerza", "poder", "violencia" (Corominas, 1980).

Otras definiciones orientan a que las violencias tienen una carga de carácter filosófico sobre la condición humana. Es un concepto complejo que se ha diversificado y tiene connotaciones de diferente orden en el espacio y/o contexto en el que se define, y por consecuencia se visualizan sus expresiones, manifestaciones, así como sus causas y efectos.

Se piensa que es imposible llegar a una única respuesta que dé cuenta en toda su magnitud del fenómeno como tal. Se ha intentado definir, conocer, estudiar, comprender, explorar e interpretar desde varias disciplinas; lo que es un hecho es que no puede analizarse en forma lineal. Se da en lo político, social, familiar, escolar; en lo deportivo: en todos los ámbitos donde el espíritu humano se despliega aparece como componente colateral, la violencia. De ahí que el fenómeno como tal, también pueda caracterizarse y dimensionarse como la transgresión a los derechos humanos y sociales fundamentales, inherentes a la naturaleza humana.

La violencia atenta contra la libertad y los dere $\urcorner$ chos humanos. Provoca daños, sufrimiento o muerte en cada mujer que la padece. Se identifican variadas expresiones de violencia hacia la mujer: la violencia que viven las migrantes; la violencia conyugal (malos tratos, violencia psicológica, económica) y que incluye en algunos casos, la violencia sexual; la violencia contra las niñas y las adultas mayores; contra las que sufren algún tipo de discapacidad; la violencia que significa la calle para las mujeres; la prostitución; la pornografía; la trata y el tráfico de personas; la violencia política y jurídica que restringe su ciudadanía plena; la que es producto de la feminización de la pobreza (las mujeres son la más pobres entre los pobres); aquella que genera víctimas de sufrimientos innecesarios como resultado de conductas negligentes o abusivas durante la provisión de servicios de salud en los que se les niega una atención adecuada, que en muchos casos termina con la muerte; la violencia estructural, entendida como las formas de violencia y desigualdad generadas por las estructuras sociales, es decir, las desigualdades entre individuos, grupos y sociedades que impiden a las personas satisfacer sus necesidades fundamentales, materiales y espirituales.

Se dice que la violencia contra las mujeres tiene su origen en algunos de las siguientes circunstancias:

- Las relaciones sociales de dominación masculina y subordinación femenina: unos mandan más que otros en la sociedad.

- La construcción de identidades de género desiguales: las identidades femeninas son menos valoradas que las masculinas. 


\section{- Senta}

- La división social del trabajo: las mujeres dedicadas al cuidado y responsabilidades familiares y los varones, a los trabajos productivos.

- La persistencia de relaciones patriarcales en la esfera familiar: los hombres siempre mandan.

A partir de los tipos de violencia que se denuncian según las instancias oficiales, se retoman datos que han sido retrabajados y que pueden explicitar la situación de violencia generalizada que padecen las mujeres y que indudablemente trastocan toda la esfera biopsicosocial que trasciende el ámbito de la mujer, es decir, impacta directamente a la sociedad en su conjunto.

\begin{tabular}{ll}
\hline \multicolumn{1}{c}{ Tipo de violencia } & \multicolumn{1}{c}{ Manifestaciones y/o características } \\
\hline Física & $\begin{array}{l}\text { Bofetadas, puñetazos, puntapiés, empujones, intento de estrangulación, } \\
\text { etcétera. }\end{array}$ \\
\hline Psicológica & Palabras hirientes, descalificaciones, humillaciones, gritos e insultos. \\
a) Amenazas & - Secuestro de los hijos, abandono, retirar el sustento económico; \\
b) Intimidación & $\begin{array}{l}\text { Miradas, acciones, gestos, destrozo de objetos personales, } \\
\text { chantaje, ostentar armas, etcétera. }\end{array}$ \\
c) Desvalorización & $\begin{array}{l}\text { Culpabilizar, humillar, insultar con apodos ofensivos, generar } \\
\text { confusión, desacreditación, etcétera. }\end{array}$
\end{tabular}

Verbal

Gritos, desprecios, insultos, mentiras, comentarios sarcásticos, burlas que exponen a la víctima en público, etcétera.

Sexual

Prácticas sexuales sin el consentimiento de las personas involucradas: sexo anal, oral, colectivo, etcétera.

Abandono

Acto de desamparo injustificado hacia uno o varios miembros de la familia con los que se tienen obligaciones que derivan de las disposiciones legales y que ponen en peligro la salud.

\section{Económica}

Impedir que la mujer trabaje o que mantenga su empleo.

No aportar al sustento de la familia.

No informar el monto de los ingresos familiares.

Obligar a algún miembro de la familia a pedir dinero.

Destruir objetos de valor.

Disponer sin consentimiento del dinero y de los bienes inmuebles que forman parte del patrimonio familia.

Fuente: elaboración propia con datos del Semáforo Delictivo del Estado de Sonora. 


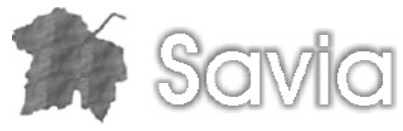

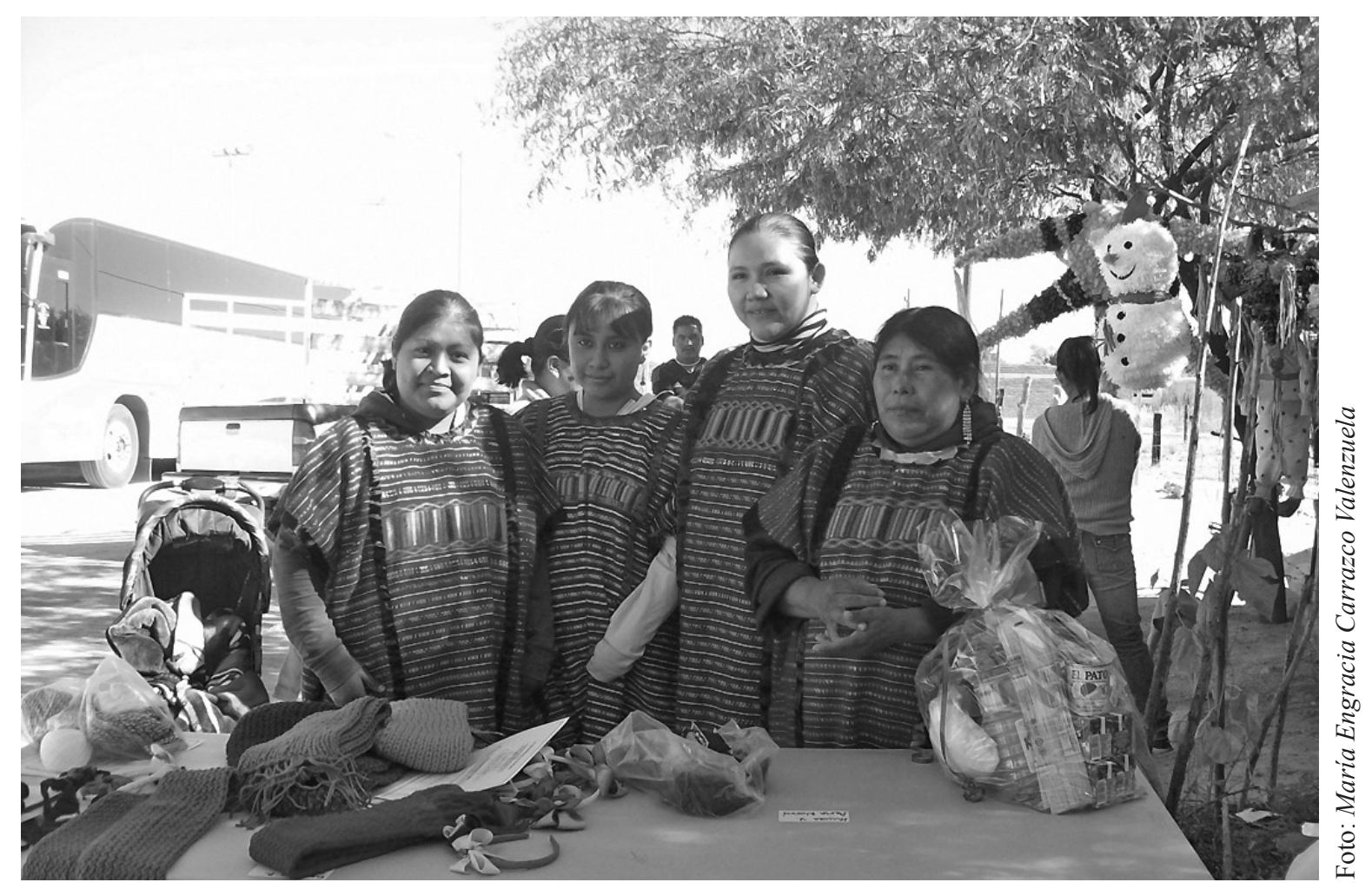

Las violencias crean desigualdad de todo tipo y sin duda, tienen una explicación desde lo cultural, ya que incorporan toda una serie de códigos y significados de una determinada cultura, que a su vez las legitiman y las hacen parecer como un medio aceptable para responder al conflicto. Es una forma en la cual un grupo de individuos o comunidad se ven en relación a sí mismos, a otros y al mundo. Frecuentemente, legitiman un sentimiento de superioridad y de menosprecio hacia otras culturas.

Martínez (2012), uno de los principales teóricos contemporáneos sobre el conflicto y la paz, hace un abordaje estructural de la violencia y afirma que la violencia no es natural a las personas, como proponen algunos autores y tradiciones filosóficas, y dice que tampoco es una afrenta "inevitable" contra la vida; expresa que se ha de buscar sus raíces en el contexto, en el modelo social, en las estructuras económicas y políticas, en las formas de abordar los conflictos y en las condiciones que favorecen el potencial para ejercerla.
Cuando se alude a la violencia de género, es necesario distinguir dos conceptos que son importantes para comprender el problema, se trata del sexo y el género. Sexo se refiere a las características biológicas y físicas que traemos al nacer y que nos definen como un hombre o una mujer. Género son aquellas características culturales que establecen las conductas consideradas propias de lo femenino y lo masculino, y de las relaciones entre ambos; relación que está caracterizada por la subordinación de un género sobre otro, es decir, por la superioridad de lo masculino sobre lo femenino.

En el sentido anterior, interesa ubicar la violencia de género y en concreto algunas reflexiones sobre la violencia contra la mujer indígena. "Las historias y la vida cotidiana de las mujeres indígenas demuestran la necesidad de contar con un punto de vista teórico que reconozca la casi universalidad de la violencia contra las mujeres y las particularidades de las experiencias de las mujeres indígenas" (FIMI, 2006:15). 


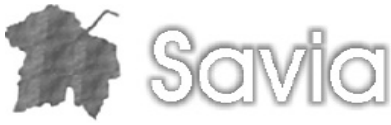

El Foro Internacional de Mujeres Indígenas, se sustenta en el marco de los derechos humanos a partir de dos principios básicos: la universalidad y la indivisibilidad de los derechos. El primero refiere que toda mujer en el mundo puede ejercer una serie de derechos sin excepciones basadas en la cultura, tradición o religión; el segundo principio considera que las mujeres indígenas solo pueden disfrutar del derecho a una vida sin violencia cuando los derechos colectivos de sus pueblos son respetados. Históricamente los grupos de población indígena han estado sujetos y han sufrido la discriminación y exclusión; las mujeres indígenas han sido protagonistas centrales en estos escenarios y se han convertido en víctimas de las acciones de violencia ejercidas en la sociedad contemporánea.

En este artículo se intenta socializar algunos de los elementos presentes en un tema que es y seguirá siendo objeto de estudio, de reflexión y análisis, para poder dimensionar las violencias en el espacio de la vida cotidiana de las mujeres y en particular, las mujeres indígenas que cada día han tratado de ganar espacios en los mundos donde viven y se desarrollan.

\section{Estación Pesqueira: una comunidad multicultural de jornaleras y jornaleros agrícolas migrantes \\ Contextualización}

La localidad de Estación Pesqueira es una comisaría perteneciente al municipio de San Miguel de Horcasitas. Está ubicada sobre la carretera libre paralela a la carretera federal número 15 y a 7 kilómetros en su acceso al corredor económico. Está aproximadamente a 40 kilómetros de la ciudad de Hermosillo, capital del estado de Sonora. La comunicación terrestre para acceder es mediante la carretera pavimentada de dos carriles que parte de San Pedro el Saucito rumbo al sur.

Se comunica con San Miguel de Horcasitas (cabecera municipal) mediante un camino de terracería de aproximadamente $30 \mathrm{~km}$, y es atravesada por tres arroyos, además del río San Miguel y corrientes superficiales que en épocas de lluvia dificultan el tránsito de personas y de automóviles.
Por estar ubicada en un camino de libramiento de la caseta de peaje, la localidad cuenta con una afluencia de automóviles y tráileres que viajan hacia el norte y sur del estado, y que deciden no pagar el peaje en la caseta de cobro. Ese tránsito permanente ha posibilitado que se establezcan pequeños negocios informales.

Tiene una mancha poblacional de familias indígenas venidas de diferentes estados de la República Mexicana, entre los que destacan: Oaxaca, Guerrero, Veracruz, Chiapas, Chihuahua, México, Michoacán, Sinaloa, Guanajuato. Hay individuos pertenecientes fundamentalmente a grupos étnicos tales como: triquis, mixtecos, zapotecos, náhuatl, tarahumaras y mayos del estado de Sonora. Estos grupos de población indígena son migrantes asentados, es decir, han emigrado de sus lugares de origen en búsqueda de trabajo y de mejores condiciones de vida. De igual manera, se identifican migrantes en tránsito temporal que llegan y se establecen en el área poblacional, solo en los periodos o épocas de pizca (cosecha) y se contratan como jornaleras y jornaleros agrícolas.

Este grupo cuenta actualmente con un promedio aproximado de 2000 personas, entre los más representativos están los zapotecos, triquis y mixtecos; en cantidades menores se encuentran las personas que representan a los otros grupos étnicos. La distribución porcentual por sexos, aproximadamente se identifica en $60 \%$ de mujeres y $40 \%$ de hombres, respectivamente (Carrasco y pérez, 2012)

\section{El caso de las mujeres indígenas triqui y la violencia de género}

Con base en los datos anteriores, en promedio existen 1020 mujeres indígenas aproximadamente; del total, 450 son niñas, adolescentes y adultas triqui, que han emigrado del estado de Oaxaca, en búsqueda de mejores condiciones de vida familiar. Cabe señalar que en el caso de las niñas triqui, han nacido en la comunidad de Pesqueira o bien, en la localidad de Hermosillo, Sonora.

Con el propósito de recuperar la información, que de viva voz se expresa a través de la técnica 


\section{就 Scivi[a]}

de entrevista a una mujer que forma parte del grupo poblacional que en líneas arriba se describe, se transcriben fragmentos que pueden ilustrar el sentir y el pensar de la identidad de una mujer indígena que dimensiona la cuestión de las violencias desde su propia perspectiva cultural y quien ha tenido la oportunidad de salir de su contexto cotidiano y haber llegado al espacio universitario.

Lo anterior permite identificar algunos de los patrones culturales que giran alrededor del ámbito familiar y de las formas de violencia que se asumen vinculadas a la condición de la mujer y su entorno.

Desde muy temprana edad, la mujer triqui es educada en su hogar a ser sumisa, obediente; no manifestar sus molestias, preocupaciones; realizar todos los deberes del hogar, cuidar a sus hermanos, con la finalidad de que cuando sea adulta y llegue al matrimonio, esté preparada a ser la mujer digna para el hombre que la venga a pedir a cambio de un dote; es decir, culturalmente las familias triqui, entregan a las hijas mujeres a cambio de bienes materiales y/o económicos, lo que formaliza la relación de matrimonio para toda la vida, pero aun más la entrega está condicionada a que la mujer sea "virgen". En caso de que la mujer hubiera tenido relaciones sexuales con otro hombre y haya sido entregada a quien la pide, el hombre se adjudica el derecho de poder tener

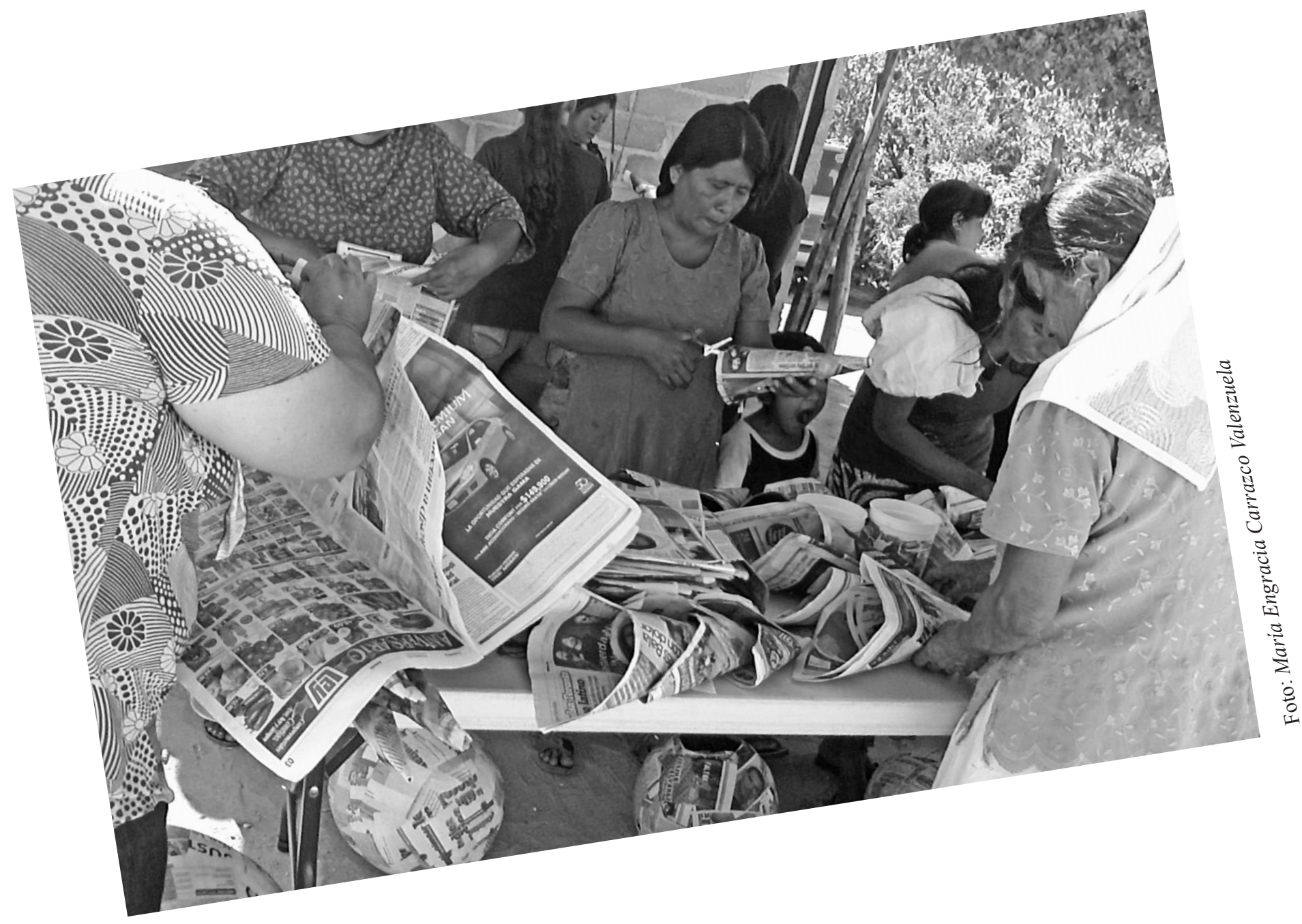




\section{霜 Sonte}

una nueva pareja sin dejar a la mujer llamada "indigna", lo que la coloca en una doble sumisión, ya que el hombre la utiliza y la explota, debido a que le corresponde salir a realizar las labores en el campo, y la nueva pareja se dedica a las tareas domésticas. Las dos mujeres pueden tener hijos del mismo hombre y quien asume las tareas del hogar tiene la obligación de cuidar a los hijos de ambas.

En el escenario anterior, se presentan graves situaciones de violencia hacia la mujer indígena triqui; más si es considerada "indigna", eso le quita cualquier derecho como mujer y el hombre ejerce acciones de violencia que van desde gritos, insultos, golpes, descalificación, rechazo, indiferencia, abandono, explotación sexual y económica, entre otras graves acciones. En la cultura triqui, las mujeres son educadas para cumplir un rol de sumisión que no les permite fácilmente salir del entorno cultural en el que han vivido durante muchas generaciones.

El hombre triqui, como jefe de familia y figura de autoridad máxima, es el único que asume el papel para "disciplinar" a las niñas y mujeres adultas de la familia, castigándolas físicamente cuando no cumplen con las obligaciones de servicio y obediencia que les asigna. Cabe mencionarse que la mujer triqui es víctima de violencia desde que crece en el espacio familiar, ya que está condicionada solo por las reglas del hombre (padres, hermanos, esposos, autoridades tradicionales).

Esto sin duda, son formas de violencia ejercidas contra la mujer, que responden a patrones socioculturales que se han convertido en prácticas aceptadas como naturales, ya que están dadas por usos y costumbres ancestrales que han permitido también la invisibilidad y silenciamiento de las mujeres indígenas.

Haber logrado una visión distinta de la condición de ser mujer a la que tradicionalmente tienen otras mujeres, ya que la educación se convierte en un recurso muy significativo para llegar a comprender la vida y el mundo desde otra perspectiva cultural.

Que cualquier acto de violencia ejercida hacia las mujeres debe ser denunciada ante las instancias correspondientes, y sensibilizar a todas aquellas mujeres que forman parte de los grupos étnicos que viven en las comunidades alejadas, ya que es en estos espacios donde cotidianamente las mujeres se convierten en víctimas del problema, y por consecuencia, las limita a llevar una vida libre de violencia.

Este artículo aporta elementos que puedan servir para la reflexión, comprensión e interpretación de un problema social que en nuestro país y particularmente en el estado de Sonora, día a día va en aumento, ya que el problema de las violencias en lo general y la violencia ejercida hacia las mujeres en lo particular, aumentan de manera exponencial.

Es de suma importancia que las profesiones que generan procesos de intervención social, tal es el caso del trabajo social, contribuyan a definir estrategias que coadyuven en acciones de prevención y atención a un problema de orden social que demanda ser intervenido desde la perspectiva de la profesión.

\section{A manera de conclusión}

Consideramos que es necesario implementar una serie de acciones encaminadas al empoderamiento de la mujer indígena, a través de programas con perspectiva de género que tengan como propósito fundamental instruir y educar para que las mujeres indígenas logren una vida más digna y justa, en los entornos comunitarios.

Las universidades públicas deben jugar un papel protagónico en tanto pueden ser impulsoras de proyectos de vinculación social, que realmente trasciendan el ámbito de la vida institucional y capitalicen sus recursos hacia la contribución de acciones sociales que vayan dirigidas a las mujeres que son un grupo de población en espera de nuevas oportunidades para avanzar en sus procesos de desarrollo.

Pensar como mujeres y como mujeres indígenas, es pensar en las oportunidades de una atención más integral donde las necesidades básicas puedan ser atendidas, ya que en este aspecto también se generan ejercicios de violencia que atentan contra las ellas cuando las demandas 


\section{- Sorvio}

no son atendidas por las instituciones responsables de los servicios de salud, educación, asistencia social, recreación y cultura, derechos humanos, sociales y laborales, etcétera.

\section{Bibliografía}

Carrazco Valenzuela, María Engracia y Bernardo Pérez García. (2012). Bienestar social para las familias indígenas asentadas en Estación Pesqueira, Mpio. de San Miguel de Horcasitas en el estado de Sonora. Documento de trabajo. Hermosillo, Sonora, México.

Carrazco Valenzuela, María Engracia y María de los Ángeles Ruiz Hernández. (2013). Patrones de discriminación y exclusión vigentes en el contexto social del municipio. En: Carlos Germán Palafox Moyers (coord.). La Violencia y la construcción del espacio local. Un enfoque multidisciplinario. El caso de Navojoa, Sonora, México. (pp. 239-264). México: Pearson.

Castro, Roberto y Florinda Riquer. (2012). Estudio Nacional sobre las Fuentes, Orígenes y Factores que Producen y Reproducen la Violencia Contra las Mujeres. Presentación y Síntesis de Resultados. Extraído en octubre de 2013, de: http://www.conavim.gob.mx/work/ models/CONAVIM/

Consejo Nacional para Prevenir la Discriminación. CONAPRED. Extraído en julio de 2013, de: http://www.conapred.org.mx/movil_tablet/ index.php

Corominas, Joan. (1980). Diccionario crítico etimológico castellano e hispánico. España: Gredos.

Entrevista. (2013). Realizada a María, mujer indígena triqui. Estación Pesqueira, Municipio de San Miguel Horcasitas.

Foro Internacional de Mujeres Indígenas (FIMI). (2006). Mujeres indígenas confrontan la violencia. Informe complementario al estudio sobre violencia contra las mujeres del Secretario General de las Naciones Unidas. Extraído en agosto de 2013, de: http://www.fimi-iiwf.org/arc hivos/8162f56478b8433333dc95a1f5f381ab1.pdf
Instituto Nacional de las Mujeres. (2013). Estadísticas de Violencia contra las Mujeres en México. Extraído en julio de 2013, de: http://estadistica.inmujeres. gob.mx/formas/convenciones/Nota.pdf

Instituto Nacional de las Mujeres. (2009). Evaluación de las medidas aplicadas para prevenir, atender, sancionar y erradicar la violencia contra las mujeres.

Extraído en julio de 2013, de:http://inmujeres.gob.mx/ index.php/biblioteca-digital/cuadernosgenero

Martínez Turcios, Luis Mario. (2012). Elementos para comprender la violencia. En: Juan Cristóbal Aldana Alfaro. Análisis interdisciplinario de las diversas formas de violencia contra la mujer. Guatemala: Cara Parens. (pp. 3-14).

Oficina de la O.N.U. para la Coordinación de asuntos humanitarios. (2005). La Violencia contra las Mujeres Expuestas. Extraído en agosto de 2013, de: http://www.irinnews.org/indepthmain.aspx? IndepthId $=59 \&$ ReportId $=72831$

Organización Panamericana de la Salud. (2002). Informe Mundial sobre la Violencia y la Salud: Resumen. Extraído en julio de 2013, de: http:// www.who.int/violence_injury_prevention/ violence/world_report/en/summary_es.pdf

Palafox Moyers, Germán (coord.). (2013). La violencia y la construcción del espacio local; un enfoque multidisciplinario. El caso de Navojoa, Sonora, México. México: Pearson.

Schmukler, Beatriz. (coord.). (1998). Familias y relaciones de género en transformación. México: EDAMEX.

Tepichin Valle, Ana María. (2008). Ciudadanía de las Mujeres y Políticas Públicas en México: Una Reflexión desde los Estudios de Género. En: Género, Cultura y Sociedad. Extraído en julio de 2013, de: http://www.academia. edu/2189795/ciudadania_de_las_mujeres_y_ Politica_PuBlica_en_mexico_una_reflexion desde_los_estudios_de_genero

UNICEF. (2005). Directrices interinstitucionales para Intervenciones contra la Violencia por Razón de Género en Situaciones Humanitarias. Extraído en agosto de 2013, de: http://www. unicef.org/spanish/emerg/index_33202.html 